\title{
CORRIGENDUM
}

\section{The prognostic impact of general and abdominal obesity in peripheral arterial disease}

\author{
G Giugliano, G Brevetti, E Laurenzano, L Brevetti, R Luciano and M Chiariello
}

International Journal of Obesity (2010) 34, 406; doi:10.1038/ijo.2009.287

Correction to: International Journal of Obesity (2010) 34, 280-286; doi:10.1038/ijo.2009.244; published online 24 November 2009

After the publication of the above article, the authors noticed a mistake in the second sentence of the paragraph 'Clinical implications'. The correct sentence should read:
However, in the REACH registry, ${ }^{39}$ the prevalence of abdominal obesity in PAD patients was as high as $44.1 \%$ and that of general obesity $23.8 \%$ - percentages only slightly lower than in coronary or cerebrovascular disease patients, which confirm the considerable burden of this condition also in PAD.

The authors would like to apologize for this mistake. 